\title{
Combinatorial Laplacian of the Matching Complex
}

\author{
Xun Dong* \\ School of Mathematics \\ University of Minnesota, Minneapolis, MN 55455 \\ xdong@math . umn.edu \\ Michelle L. Wachs ${ }^{\dagger}$ \\ Department of Mathematics \\ University of Miami, Coral Gables, FL 33124 \\ wachs@math.miami.edu
}

Submitted: September 28, 2000; Accepted: April 2, 2002.

MR Subject Classifications: Primary 05E10, 05E25; Secondary 05E05, 20C30, 55U10.

\begin{abstract}
A striking result of Bouc gives the decomposition of the representation of the symmetric group on the homology of the matching complex into irreducibles that are self-conjugate. We show how the combinatorial Laplacian can be used to give an elegant proof of this result. We also show that the spectrum of the Laplacian is integral.
\end{abstract}

\section{Introduction}

The matching complex of a graph $G$ is the abstract simplicial complex whose vertex set is the set of edges of $G$ and whose faces are sets of edges of $G$ with no two edges meeting at a vertex. The matching complex of the complete graph (known simply as the matching complex) and the matching complex of the complete bipartite graph (known as the chessboard complex) have arisen in a number of contexts in the literature (see eg. [6] [16] [2] [19] [3] [4] [8] [12] [1] [9] [15] [13] [17] [18]). Closely related complexes have been considered in [7] and [14].

Let $M_{n}$ denote the matching complex of the complete graph on node set $\{1, \ldots, n\}$. The symmetric group $\mathfrak{S}_{n}$ acts on the matching complex $M_{n}$ by permuting the graph

* Research supported in part by a University of Minnesota McKnight Land Grant Professorship held by Victor Reiner. Current address: Department of Mathematics, Caltech, Pasadena, CA 91125.

${ }^{\dagger}$ Research supported in part by NSF grant DMS 9701407. 
nodes. This induces a representation on the reduced simplicial homology $\tilde{H}_{r}\left(M_{n} ; k\right)$, where throughout this paper $k$ is a field of characteristic 0. The Betti numbers for the matching complex and the decomposition of the representation into irreducibles were computed by Bouc [3], and later independently by Karaguezian [8] and by Reiner and Roberts [12] as part of a more general study. They prove the following result.

Theorem 1.1 (Bouc [3]). For all $r \geq 1$ and $n \geq 2$, the following isomorphism of $\mathfrak{S}_{n}$-modules holds

$$
\tilde{H}_{r-1}\left(M_{n} ; k\right) \cong \mathfrak{S}_{n} \bigoplus_{\substack{\lambda: \lambda \vdash n \\ \lambda=\lambda^{\prime} \\ d(\lambda)}=n-2 r} S^{\lambda}
$$

where $S^{\lambda}$ denotes the Specht module indexed by $\lambda, \lambda^{\prime}$ denotes the conjugate of $\lambda$ and $d(\lambda)$ denotes the size of the Durfee square of $\lambda$.

Józefiak and Weyman [7] and Sigg [14] independently obtained an equivalent result for a Koszul complex of $G L(n, k)$-modules (cf. [9]). They use this to give representation theoretic interpretations of the following classical symmetric function identity of Littlewood

$$
\prod_{i \leq j}\left(1-x_{i} x_{j}\right) \prod_{i}\left(1+x_{i}\right)^{-1}=\sum_{\lambda=\lambda^{\prime}}(-1)^{\frac{|\lambda|+d(\lambda)}{2}} s_{\lambda} .
$$

Using Theorem 1.1 one can interpret Littlewood's formula as the Hopf trace formula for the matching complex. This interpretation is essentially equivalent to Sigg's interpretation.

A decomposition for the chessboard complex analogous to Theorem 1.1 was obtained independently by Friedman and Hanlon [4] and later by Reiner and Roberts [12] in greater generality. The method of Friedman and Hanlon [4] is particularly striking. It involves the combinatorial Laplacian which is an analogue of the Laplacian on differential forms for a Riemannian manifold. The analogue of Hodge theory states that the kernel of the combinatorial Laplacian is isomorphic to the homology of the complex. By analyzing the action of the Laplacian on oriented simplexes and applying results from symmetric function theory, Friedman and Hanlon are able to decompose all the eigenspaces of the Laplacian into irreducibles and thereby decompose the homology. They also show that the spectrum of the Laplacian is integral.

The aim of this note is to work out analogous decompositions for the combinatorial Laplacian on the matching complex. This results in an elegant proof of Theorem 1.1 which is given in Section 3. Our key observation is that the Laplacian operator behaves as multiplication by a certain element in the center of the group algebra, namely the sum of all transpositions in $\mathfrak{S}_{n}$. We also establish integrality of the spectrum of the Laplacian in Section 3.

Sigg [14] uses the Lie algebra Laplacian to obtain equivalent decompositions for the Lie algebra homology of the free two-step nilpotent complex Lie algebra. Sigg works within the framework of representation theory of the Lie algebra $g l_{n}$ and expresses the Laplacian in terms of the Casimir operator. Our approach parallels his, but at a more elementary 
level, making use of readily available facts from symmetric group representation theory. In Section 4 we establish the equivalence of our results to Sigg's results.

Reiner and Roberts [12] generalize Theorem 1.1 to general bounded degree graph complexes by using techniques from commutative algebra. In [9] it is shown that one can derive the Reiner-Roberts result from Theorem 1.1 by taking weight spaces of the $G L_{n^{-}}$ modules considered by Józefiak and Weyman. Hence, although the Laplacian technique does not appear to be directly applicable to general bounded degree graph complexes, the Laplacian provides an indirect path to the Reiner-Roberts result (and the JózefiakWeyman result) that is considerably simpler than the earlier approaches.

\section{The Combinatorial Laplacian}

Let $\Delta$ be a finite simplicial complex on which a group $G$ acts simplicially. For $r \geq-1$, let $C_{r}(\Delta)$ be the $r$ th chain space (with coefficients in $k$ ) of $\Delta$. That is, $C_{r}(\Delta)$ is the $k$-vector space generated by oriented simplexes of dimension $r$. Two oriented simplexes are related by

$$
\left(v_{1}, v_{2}, \ldots, v_{r+1}\right)=\operatorname{sgn} \sigma\left(v_{\sigma(1)}, v_{\sigma(2)}, \ldots, v_{\sigma(r+1)}\right),
$$

where $\sigma \in \mathfrak{S}_{r+1}$. The simplicial action of $G$ induces a representation of $G$ on the vector space $C_{r}(\Delta)$.

The boundary map

$$
\partial_{r}: C_{r}(\Delta) \rightarrow C_{r-1}(\Delta)
$$

is defined on oriented simplexes by

$$
\partial_{r}\left(v_{1}, \ldots, v_{r+1}\right)=\sum_{j=1}^{r+1}(-1)^{j}\left(v_{1}, \ldots, \hat{v}_{j}, \ldots, v_{r+1}\right) .
$$

Since $\partial_{*}$ commutes with the action of $G$ on $C_{*}(\Delta),\left(C_{r}(\Delta), \partial_{r}\right)$ is a complex of $G$-modules. It follows that the (reduced) homology groups $\tilde{H}_{r}(\Delta ; k)$ are $G$-modules.

The coboundary map

$$
\delta_{r}: C_{r}(\Delta) \rightarrow C_{r+1}(\Delta)
$$

is defined by

$$
\left\langle\delta_{r}(\alpha), \beta\right\rangle=\left\langle\alpha, \partial_{r+1}(\beta)\right\rangle,
$$

where $\alpha \in C_{r}(\Delta), \beta \in C_{r+1}(\Delta)$ and $\langle$,$\rangle is the bilinear form on \oplus_{r=-1}^{d} C_{r}(\Delta)$ for which any basis of oriented simplexes is orthonormal. Note that the action of $G$ on $C_{r}(\Delta)$ respects the form $\langle$,$\rangle and commutes with the coboundary map. Hence \left(C_{r}(\Delta), \delta_{r}\right)$ is a complex of $G$-modules.

The combinatorial Laplacian is the G-module homomorphism

$$
\Lambda_{r}: C_{r}(\Delta) \rightarrow C_{r}(\Delta)
$$

defined by

$$
\Lambda_{r}=\delta_{r-1} \partial_{r}+\partial_{r+1} \delta_{r}
$$


Although the following analogue of Hodge theory is usually stated and easily proved for $k=\mathbb{R}$ or $k=\mathbb{C}$ (cf. [4, Proposition 1], [14, Proposition 9], [10]), the universal coefficient theorem enables one to prove it for general fields $k$ of characteristic 0 . Indeed, one uses the universal coefficient theorem first to derive the result for $k=\mathbb{Q}$ from the result for $k=\mathbb{C}$ and then to go from $k=\mathbb{Q}$ to general fields of characteristic 0 .

Proposition 2.1. For all $r$, the following $k G$-module isomorphism holds:

$$
\operatorname{ker} \Lambda_{r} \cong_{G} \tilde{H}_{r}(\Delta ; k) .
$$

\section{Spectrum of the Laplacian and Bouc's Theorem}

The notation used here comes from [11]. The plethysm or composition product of a $\mathfrak{S}_{m^{-}}$ module $V$ and a $\mathfrak{S}_{n}$-module $U$ is the $\mathfrak{S}_{m n}$-module denoted by $V \circ U$. The induction product of $U$ and $V$ is the $\mathfrak{S}_{m+n}$-module denoted by $U . V$.

Proposition 3.1. For all $r \geq 1$ and $n \geq 2$ we have the following isomorphism of $\mathfrak{S}_{n^{-}}$ modules

$$
C_{r-1}\left(M_{n}\right) \cong_{\mathfrak{S}_{n}}\left(S^{1^{r}} \circ S^{2}\right) \cdot S^{n-2 r} .
$$

Proof. Straight forward observation.

We say that a partition is almost self-conjugate if it is of the form $\left(\alpha_{1}+1, \ldots, \alpha_{d}+1 \mid\right.$ $\left.\alpha_{1}, \ldots, \alpha_{d}\right)$ in Frobenius notation.

Proposition 3.2 (Littlewood, cf. [11, I 5 Ex. 9b]). For all $r \geq 1$,

$$
S^{1^{r}} \circ S^{2} \cong_{\mathfrak{S}_{2 r}} \bigoplus_{\lambda} S^{\lambda}
$$

summed over all almost self-conjugate partitions $\lambda \vdash 2 r$.

Pieri's rule and the fact that the induction product is linear in each of its factors yields the following.

Proposition 3.3. For all $r \geq 1$ and $n \geq 2$ we have

$$
C_{r-1}\left(M_{n}\right) \cong_{\mathfrak{S}_{n}} \bigoplus_{\substack{\lambda \in \mathcal{A} \\|\lambda|=n}} a_{\lambda}^{r} S^{\lambda},
$$

where

$$
\mathcal{A}=\left\{\left(\alpha_{1}, \ldots, \alpha_{d} \mid \beta_{1}, \ldots, \beta_{d}\right) \mid d \geq 1, \quad \alpha_{i} \geq \beta_{i} \forall i\right\}
$$

and $a_{\lambda}^{r}$ is the number of almost self-conjugate partitions $\mu \vdash 2 r$ such that $\lambda / \mu$ is a horizontal strip. 
Proposition 3.4. Let $\lambda \vdash n$ be self-conjugate. Then

$$
a_{\lambda}^{r}= \begin{cases}1 & \text { if } d(\lambda)=n-2 r \\ 0 & \text { otherwise }\end{cases}
$$

Proof. Straight forward observation.

Propositions 3.1, 3.3 and 3.4 comprise the first steps of Bouc's proof of Theorem 1.1. At this point our proof departs from Bouc's and follows a path analogous to that of Friedman and Hanlon [4] for the chessboard complex.

Consider the element $T_{n}=\sum_{1 \leq i<j \leq n}(i, j)$ of $k \mathfrak{S}_{n}$, where $(i, j)$ denotes a transposition in $\mathfrak{S}_{n}$. In any $\mathfrak{S}_{n}$-module $M$, left multiplication by $T_{n}$ is an endomorphism of the $\mathfrak{S}_{n^{-}}$ module, since $T_{n}$ is in the center of $k \mathfrak{S}_{n}$. We will denote this endomorphism by $T_{M}$. The Laplacian will be denoted by $\Lambda_{n, r}: C_{r}\left(M_{n}\right) \rightarrow C_{r}\left(M_{n}\right)$.

Lemma 3.5. For all $r \geq 1$ and $n \geq 2$,

$$
\Lambda_{n, r-1}=T_{C_{r-1}\left(M_{n}\right)}
$$

Proof. It is a routine exercise to check that $\Lambda_{n, r-1}(\gamma)=T_{n} \cdot \gamma$ for the oriented $(r-1)$ simplex $\gamma=(\{1,2\}, \ldots,\{2 r-1,2 r\})$ which generates the $\mathfrak{S}_{n}$-module $C_{r-1}\left(M_{n}\right)$.

Proposition 3.6 (Friedman and Hanlon [4, Lemma 1]). For all $\lambda \vdash n$,

$$
T_{S^{\lambda}}=c_{\lambda} \operatorname{id}_{S^{\lambda}}
$$

where $c_{\lambda}=\sum(i-1) \lambda_{i}^{\prime}-\sum(i-1) \lambda_{i}$.

Proof. This follows from Schur's lemma and from I7, Example 7 of [11].

Lemma 3.7. For any partition $\lambda=\left(\alpha_{1}, \ldots, \alpha_{d} \mid \beta_{1}, \ldots, \beta_{d}\right)$ we have

$$
c_{\lambda}=\sum_{i=1}^{d}\left(\left(\begin{array}{c}
\alpha_{i}+1 \\
2
\end{array}\right)-\left(\begin{array}{c}
\beta_{i}+1 \\
2
\end{array}\right)\right) \text {. }
$$

Proof. Easy.

Theorem 3.8. All the eigenvalues of the Laplacian on the matching complex are nonnegative integers. Moreover for each eigenvalue $c$, the c-eigenspace of $\Lambda_{n, r-1}$ decomposes into the following direct sum of irreducibles

$$
\bigoplus_{\substack{\lambda \in \mathcal{A} \\|\lambda|=n \\ c_{\lambda}=c}} a_{\lambda}^{r} S^{\lambda},
$$

where $\mathcal{A}$ and $a_{\lambda}^{r}$ are as in Proposition 3.3. 
Proof. Proposition 3.3, Lemma 3.5 and Proposition 3.6 imply that each eigenvalue c is an integer and yield the decomposition of the $c$-eigenspace. It is immediate from Lemma 3.7 that $c_{\lambda} \geq 0$ for all $\lambda \in \mathcal{A}$. Hence there can be no negative eigenvalues. One can also conclude that the eigenvalues are nonnegative by using the fact that all rational eigenvalues of the Laplacian (over any field of characteristic 0) of any simplicial complex are nonnegative. This fact follows from the positive semidefinitness of the Laplacian over $\mathbb{C}$.

Proof of Bouc's Theorem. It follows from Lemma 3.7 that if $\lambda \in \mathcal{A}$ then $c_{\lambda}=0$ if and only if $\lambda$ is self-conjugate. Hence Theorem 3.8 and Proposition 3.4 imply that the kernel of the Laplacian decomposes into

$$
\operatorname{ker} \Lambda_{n, r-1} \cong_{\mathfrak{S}_{n}} \bigoplus_{\begin{array}{c}
\lambda: \lambda \vdash n \\
\lambda=\lambda^{\prime} \\
d(\lambda)=n-2 r
\end{array}} S^{\lambda} .
$$

Bouc's Theorem now follows from Proposition 2.1.

Remark. Bouc's Theorem is stated and proved in [3] for fields of finite characteristic $p>n$ as well as for fields of characteristic 0 . The characteristic $p>n$ case follows from the characteristic 0 case provided one knows that there is no $p$-torsion in integral homology. The lack of $p$-torsion for $p>n$ follows easily from a long exact sequence of Bouc [3, Lemme 7] which is the starting point of Bouc's proof.

\section{Sigg's Lie Algebra Homology Theorem}

In [14], Sigg decomposes the homology of the free two-step nilpotent complex Lie algebra of rank $n$ into irreducible $G L(n, \mathbb{C})$-modules by using a Laplacian operator. In this section we will describe how his results relate to ours.

Let $E$ be an $n$-dimensional complex vector space, where $n \geq 2$. Let $\wedge^{r} E$ denote the $r$ th exterior power and $\wedge^{*} E$ denote the exterior algebra of $E$. The free two-step nilpotent complex Lie algebra of rank $n$ is the vector space $\wedge^{2} E \oplus E$ with Lie bracket defined on generators by

$$
[x, y]= \begin{cases}x \wedge y & \text { if } x, y \in E \\ 0 & \text { if } x \in \wedge^{2} E \text { or } y \in \wedge^{2} E .\end{cases}
$$

For each $r \in \mathbb{N}$, form the $G L(E)$-submodule

$$
V_{r}(E)=\wedge^{r}\left(\wedge^{2} E\right) \otimes \wedge^{*} E
$$

of the exterior algebra of $\wedge^{2} E \oplus E$. The map $\partial_{r}^{E}: V_{r}(E) \rightarrow V_{r+1}(E)$ defined on generators by

$$
\partial_{r}^{E}\left(f \otimes\left(e_{1} \wedge \cdots \wedge e_{t}\right)\right)=\sum_{i<j}(-1)^{i+j}\left(f \wedge\left(e_{i} \wedge e_{j}\right)\right) \otimes\left(e_{1} \wedge \cdots \wedge \hat{e}_{i} \wedge \cdots \wedge \hat{e}_{j} \wedge \cdots \wedge e_{t}\right),
$$


where $f \in \wedge^{r}\left(\wedge^{2} E\right)$ and $e_{1}, \ldots, e_{t} \in E$, is the standard Lie algebra homology differential for the Lie algebra $\wedge^{2} E \oplus E$. The complex $\left(V_{r}(E), \partial_{r}^{E}\right)$ is a complex of $G L(E)$-modules.

The adjoint map $\delta_{r}^{E}: V_{r}(E) \rightarrow V_{r-1}(E)$ (with respect to the Hermitian form for which the standard basis of $V_{r}(E)$ is orthonormal) is defined on generators by

$$
\begin{gathered}
\delta_{r}^{E}\left(\left(\left(e_{1} \wedge e_{2}\right) \wedge \cdots \wedge\left(e_{2 r-1} \wedge e_{2 r}\right)\right) \otimes e\right) \\
=\sum_{j=1}^{r}(-1)^{j+r+1}\left(\left(e_{1} \wedge e_{2}\right) \wedge \cdots \wedge\left(\widehat{e_{2 j-1} \wedge e_{2 j}}\right) \wedge \cdots \wedge\left(e_{2 r-1} \wedge e_{2 r}\right)\right) \otimes\left(e_{2 j-1} \wedge e_{2 j} \wedge e\right),
\end{gathered}
$$

where $e_{1}, \ldots, e_{2 r} \in E$ and $e \in \wedge^{*} E$. Let $H_{r}(V(E))$ denote the homology of the $G L(E)$ complex $\left(V_{r}(E), \partial_{r}^{E}\right)$ and let $H^{r}(V(E))$ denote the homology of the $G L(E)$-complex $\left(V_{r}(E), \delta_{r}^{E}\right)$.

The Laplacian used by Sigg is the $G L(E)$-homomorphism $\Lambda_{r}^{E}: V_{r}(E) \rightarrow V_{r}(E)$ defined by

$$
\Lambda_{r}^{E}=\delta_{r+1}^{E} \partial_{r}^{E}+\partial_{r-1}^{E} \delta_{r}^{E} .
$$

It follows from the discrete version of Hodge theory that

$$
H_{r}(V(E)) \cong_{G L(E)} H^{r}(V(E)) \cong_{G L(E)} \operatorname{ker} \Lambda_{r}^{E} .
$$

For each partition $\lambda$, let $E^{\lambda}$ be the irreducible polynomial representation of $G L(E)$ of highest weight $\lambda$ if $\ell(\lambda) \leq \operatorname{dim} E$ and 0 otherwise.

Proposition 4.1 (Sigg [14]). All eigenvalues of $\Lambda_{r}^{E}$ are nonnegative integers. Moreover for each eigenvalue $c$, the c-eigenspace decomposes into the following direct sum of irreducibles

$$
\bigoplus_{\substack{\lambda \in \mathcal{A} \\ c_{\lambda}=c}} a_{\lambda}^{r} E^{\lambda^{\prime}}
$$

where $\mathcal{A}$ and $a_{\lambda}^{r}$ are as in Proposition 3.3. Consequently,

$$
H_{r}(V(E)) \cong_{G L(E)} H^{r}(V(E)) \cong_{G L(E)} \bigoplus_{\substack{\lambda: \lambda=\lambda^{\prime} \\ d(\lambda)=|\lambda|-2 r}} E^{\lambda} .
$$

Sigg proves this result by first switching to the derivative representation of the Lie algebra $g l(E)$ on $\oplus_{r} V_{r}(E)$, and then comparing the Casimir operator of the $g l(E)$-module to the Laplacian operator.

We will now describe how one can obtain Sigg's result from our Proposition 3.8 and vice-versa. Let $U_{n, r}$ be the $1^{n}$-weight space of the $G L(E)$-module $V_{r}(E)$ (recall $n=\operatorname{dim} E$ ). (See [5] for information on weight spaces.) The weight space $U_{n, r}$ is a $\mathfrak{S}_{n}$-module. Since $\Lambda_{r}^{E}\left(U_{n, r}\right) \subseteq U_{n, r}$, by restricting the Laplacian we get a $\mathfrak{S}_{n}$-module homomorphism $\mathcal{L}_{n, r}$ : $U_{n, r} \rightarrow U_{n, r}$. Now we take the Young dual. That is, we consider the map

$$
\mathcal{L}_{n, r} \otimes \mathrm{id}: U_{n, r} \otimes \operatorname{sgn} \rightarrow U_{n, r} \otimes \operatorname{sgn},
$$

where sgn denotes the sign representation of $\mathfrak{S}_{n}$. 
Theorem 4.2. There is an $\mathfrak{S}_{n}$-module isomorphism $\phi_{r}: U_{n, r} \otimes \operatorname{sgn} \rightarrow C_{r-1}\left(M_{n}\right)$ such that

$$
\phi_{r} \circ\left(\mathcal{L}_{n, r} \otimes \mathrm{id}\right)=\Lambda_{n, r-1} \circ \phi_{r} .
$$

Proof. Consider the oriented $(r-1)$-simplex

$$
c=(\{1,2\}, \ldots,\{2 r-1,2 r\})
$$

in $C_{r-1}\left(M_{n}\right)$ and the element

$$
d=\left(\left(e_{1} \wedge e_{2}\right) \wedge \cdots \wedge\left(e_{2 r-1} \wedge e_{2 r}\right)\right) \otimes\left(e_{2 r+1} \wedge \cdots \wedge e_{n}\right)
$$

of $U_{n, r}$, where $e_{1}, \ldots, e_{n}$ is a fixed ordered basis for $E$. Then

$$
\left\{\sigma \cdot c \mid \sigma \in \mathfrak{S}_{n}\right\} \text { and }\left\{\sigma \cdot d \mid \sigma \in \mathfrak{S}_{n}\right\}
$$

are spanning sets for the respective vector spaces $C_{r-1}\left(M_{n}\right)$ and $U_{n, r}$.

Let $\xi$ be a generator of the one dimensional sgn representation. Define $\phi_{r}$ on the elements of the spanning set by

$$
\phi_{r}(\sigma \cdot d \otimes \xi)=\operatorname{sgn}(\sigma) \sigma \cdot c .
$$

It is easy to see that this determines a well-defined $\mathfrak{S}_{n}$-module isomorphism $\phi_{r}: U_{n, r} \otimes$ sgn $\rightarrow C_{r-1}\left(M_{n}\right)$ by checking that relations on the elements of the spanning set of the vector space $U_{n, r} \otimes \operatorname{sgn}$ correspond (under $\phi_{r}$ ) to relations on the elements of the spanning set of the vector space $C_{r-1}\left(M_{n}\right)$.

One can also easily check that

$$
\phi_{r+1}\left(\partial_{r}^{E}(d) \otimes \xi\right)=\delta_{n, r-1}\left(\phi_{r}(d \otimes \xi)\right)
$$

and

$$
\phi_{r-1}\left(\delta_{r}^{E}(d) \otimes \xi\right)=\partial_{n, r-1}\left(\phi_{r}(d \otimes \xi)\right),
$$

where $\partial_{n, r-1}$ and $\delta_{n, r-1}$ denote the $(r-1)$-boundary and $(r-1)$-coboundary maps, respectively, of the matching complex $M_{n}$. From this it follows that

$$
\phi_{r}\left(\Lambda_{r}^{E}(d) \otimes \xi\right)=\Lambda_{n, r-1}\left(\phi_{r}(d \otimes \xi)\right) .
$$

So $\phi_{r} \circ\left(\mathcal{L}_{n, r} \otimes\right.$ id $)$ and $\Lambda_{n, r-1} \circ \phi_{r}$ agree on the generator $d \otimes \xi$ of the cyclic $\mathfrak{S}_{n}$-module, and they are therefore the same map.

Corollary 4.3. The eigenvalues of $\Lambda_{n, r-1}$ and $\mathcal{L}_{n, r}$ are the same. Moreover, for each eigenvalue $c$ and partition $\lambda$ of $n$, the multiplicity of the irreducible $S^{\lambda}$ in the c-eigenspace of $\Lambda_{n, r-1}$ equals the multiplicity of $S^{\lambda^{\prime}}$ in the c-eigenspace of $\mathcal{L}_{n, r}$.

The following proposition and Corollary 4.3 establish the equivalence of Proposition 4.1 and Theorem 3.8 . 
Proposition 4.4. For all $c$ and all partitions $\lambda$ such that $\ell(\lambda) \leq \operatorname{dim} E$, the multiplicity of the irreducible $E^{\lambda}$ in the c-eigenspace of $\Lambda_{r}^{E}$ equals the multiplicity of $S^{\lambda}$ in the ceigenspace of $\mathcal{L}_{|\lambda|, r}$.

Proof. Suppose $|\lambda|=\operatorname{dim} E$. Then the $c$-eigenspace of $\mathcal{L}_{|\lambda|, r}$ is the $1^{|\lambda|}$-weight space of the $c$-eigenspace of $\Lambda_{r}^{E}$. By taking the $1^{|\lambda|}$-weight space of each summand in the decomposition of the $c$-eigenspace of $\Lambda_{r}^{E}$ into irreducible $G L(E)$-modules, we obtain a decomposition of the $c$-eigenspace of $\mathcal{L}_{|\lambda|, r}$ into irreducible $\mathfrak{S}_{|\lambda|}$-modules $S^{\lambda}$ whose multiplicity is the same as that of $E^{\lambda}$ in the $c$-eigenspace of $\Lambda_{r}^{E}$.

To obtain the result for general $\lambda$ from the case that $|\lambda|=\operatorname{dim} E$, we need only observe the fact that if $E_{1}$ and $E_{2}$ are $t$ and $s$ dimensional vector spaces, respectively, and $\lambda$ is a partition such that $\ell(\lambda) \leq t \leq s$, then the multiplicity of $E_{2}^{\lambda}$ in the $c$-eigenspace of $\Lambda_{r}^{E_{2}}$ equals the multiplicity of $E_{1}^{\lambda}$ in the $c$-eigenspace of $\Lambda_{r}^{E_{1}}$. To establish this fact, suppose $E_{1}$ has ordered basis $e_{1}, \ldots, e_{t}$ and $E_{2}$ has ordered basis $e_{1}, \ldots, e_{s}$. For any polynomial $G L\left(E_{2}\right)$-module $V$ and sequence $\mu=\left(\mu_{1}, \ldots, \mu_{s}\right)$ of nonnegative integers let $V_{\mu}$ denote the $\mu$-weight space of $V$. For $i=1,2$, let $W_{i}(c)$ be the $c$-eigenspace of $\Lambda_{r}^{E_{i}}$. Note that

$$
W_{1}(c)=\bigoplus_{\mu} W_{2}(c)_{\mu},
$$

where $\mu$ ranges over all weights $\left(\mu_{1}, \ldots, \mu_{s}\right)$ such that $\mu_{t+1}=\cdots=\mu_{s}=0$. Suppose $W_{2}(c)$ decomposes into $\bigoplus b_{\lambda} E_{2}^{\lambda}$. Then

$$
W_{2}(c)_{\mu}=\bigoplus_{\lambda} b_{\lambda}\left(E_{2}^{\lambda}\right)_{\mu}
$$

It follows that

$$
W_{1}(c)=\bigoplus_{\mu} \bigoplus_{\lambda} b_{\lambda}\left(E_{2}^{\lambda}\right)_{\mu}=\bigoplus_{\lambda} \bigoplus_{\mu} b_{\lambda}\left(E_{2}^{\lambda}\right)_{\mu}=\bigoplus_{\lambda} b_{\lambda} E_{1}^{\lambda} .
$$

Hence the multiplicities of $E_{2}^{\lambda}$ in $W_{2}(c)$ and $E_{1}^{\lambda}$ in $W_{1}(c)$ are the same.

\section{Acknowledgements}

The authors would like to thank Victor Reiner for many useful discussions. Part of the work on this paper was carried out while the second author was a guest researcher at the Royal Institute of Technology in Stockholm in 1999. The second author would like to thank the Institute and Anders Björner for their hospitality and support.

\section{References}

[1] E. Babson, A. Björner, S. Linusson, J. Shareshian and V. Welker, Complexes of not i-connected graphs, Topology 38 (1999), 271-299. 
[2] A. Björner, L. Lovász, S.T. Vrećica and R.T. Živaljević, Chessboard complexes and matching complexes, J. London Math. Soc. 49 (1994), 25-39.

[3] S. Bouc, Homologie de certains ensembles de 2-sous-groups des groupes symétriques, J. Algebra 150 (1992), 158-186.

[4] J. Friedman and P. Hanlon, On the Betti numbers of chessboard complexes, J. Alg. Comb. 8 (1998), 193-203.

[5] W. Fulton, Young Tableau, London Mathematical Society Student Texts 35, Cambridge University Press, 1997.

[6] P.F. Garst, Cohen-Macaulay complexes and group actions, Ph.D. Thesis, University of Wisconsin-Madison, 1979.

[7] T. Józefiak and J. Weyman, Representation-theoretic interpretation of a formula of D.E. Littlewood, Math. Proc. Cambridge Philos. Soc. 103 (1988) 193-196.

[8] D.B. Karaguezian, Homology of complexes of degree one graphs, Ph.D. Thesis, Stanford University, 1994.

[9] D. Karaguezian, V. Reiner, M.L. Wachs, Matching Complexes, bounded degree graph complexes and weight spaces of $G L_{n}$-complexes, preprint 1999.

[10] B. Kostant, Lie algebra cohomology and the generalized Borel-Weil theorem, Ann. Math. 74(1961), 329-387.

[11] I.G. Macdonald, Symmetric Functions and Hall Polynomials, Oxford University Press, second edition, 1995.

[12] V. Reiner and J. Roberts, Minimal resolutions and homology of chessboard and matching complexes, J. Algebraic Combinatorics 11 (2000), 135-154.

[13] J. Shareshian and M.L. Wachs, Torsion in the homology of the matching complex and chessboard complex, to appear.

[14] S. Sigg, Laplacian and homology of free two-step nilpotent Lie algebras, J. Algebra 185(1996), 144-161.

[15] S. Sundaram, On the topology of two partition posets with forbidden block sizes, to appear in J. Pure and Appl. Algebra.

[16] S.T. Vrećica and R.T. Živaljević, The colored Tverberg problem and complexes of injective functions, J. Combin. Theory Ser. A 61 (1992), 309-318.

[17] M.L. Wachs, Bounded degree digraph and multigraph complexes, to appear.

[18] M.L. Wachs, Topology of matching, chessboard, and general bounded degree graph complexes, Gian-Carlo Rota memorial issue of Algebra Universalis, to appear. 
[19] G.M. Ziegler, Shellability of chessboard complexes, Israel J. Math. 87 (1994), 97110. 